\title{
First results of total and partial cross-section measurements of the ${ }^{107} \mathrm{Ag}(\mathbf{p}, \gamma){ }^{108} \mathrm{Cd}$ reaction
}

\author{
Felix Heim ${ }^{1, \star}$, Jan Mayer ${ }^{1}$, Philipp Scholz ${ }^{1}$, Mark Spieker $^{1}$, and Andreas Zilges ${ }^{1}$ \\ ${ }^{1}$ Institute for Nuclear Physics, University of Cologne
}

\begin{abstract}
The $\gamma$ process is assumed to play an important role in the nucleosynthesis of the majority of the $p$ nuclei. Since the network of the $\gamma$ process includes so many different reactions and - mainly unstable - nuclei, cross-section values are predominantly calculated in the scope of the Hauser-Feshbach statistical model. The values heavily depend on the nuclear-physics input parameters. The results of total and partial crosssection measurements are used to improve the accuracy of the theoretical calculations. In order to extend the experimental database, the ${ }^{107} \mathrm{Ag}(\mathrm{p}, \gamma){ }^{108} \mathrm{Cd}$ reaction was studied via the in-beam method at the high-efficiency HPGe $\gamma$-ray spectrometer HORUS at the University of Cologne. Proton beams with energies between $3.5 \mathrm{MeV}$ and $5.0 \mathrm{MeV}$ were provided by the $10 \mathrm{MV}$ FN-Tandem accelerator leading to the determination of four new total cross-section values. After slight adjustments of the nuclear level density and $\gamma$-ray strength function an excellent agreement between theoretical calculations and experimentally deduced values for both total and partial cross sections has been obtained.
\end{abstract}

\section{Introduction}

About 30 to 35 neutron-deficient nuclei are bypassed by neutron-capture processes and the question about their detailed origin is still open. There are several different processes in various astrophysical scenarios proposed which might contribute to the synthesis of the so-called $p$ nuclei $[1,2]$. However, the main contribution is assumed to stem from the $\gamma$-process, a huge network of photodisintegration reactions on approx. 2,000 mainly unstable nuclei. In order to predict reaction rates or cross-section values, one often has to rely on statistical model calculations which are mostly performed in the scope of the Hauser-Feshbach statistical model. In order to reduce uncertainties and improve the predictive power of the statistical calculations, the models for nuclear-physics input parameter have to be improved. Therefore measurements in or close to the Gamow window - usually way below the Coulomb barrier - are necessary. In order to study the nuclear level density as well as the $\gamma$ ray strength function the ${ }^{107} \mathrm{Ag}(\mathrm{p}, \gamma){ }^{108} \mathrm{Cd}$ reaction was measured, for which the Gamow window is between $1.77 \mathrm{MeV}$ and $3.83 \mathrm{MeV}$ at $\mathrm{T}_{9}=3$ [3].

By a comparison between experimentally obtained results to theory, different models can either be excluded or validated. Here, preliminary experimental results on the measurements of total and partial cross-section values of the ${ }^{107} \mathrm{Ag}(\mathrm{p}, \gamma){ }^{108} \mathrm{Cd}$ reaction as well as the comparison to statistical model calculations are presented.

\footnotetext{
^e-mail: fheim@ikp.uni-koeln.de
} 


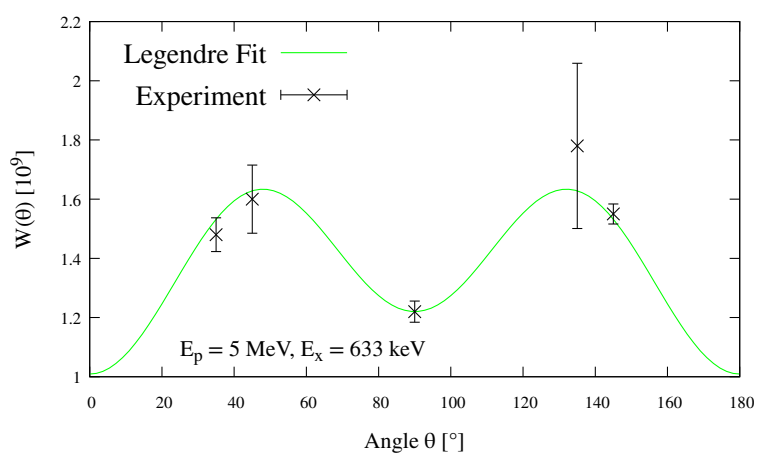

Figure 1. Angular distribution of the decay $2_{1}^{+} \rightarrow$ g.s. in ${ }^{108} \mathrm{Cd}$ for an incident beam energy of $5 \mathrm{MeV}$. For the data points a sum of Legendre polynomials is fitted in order to obtain the total number of reactions.

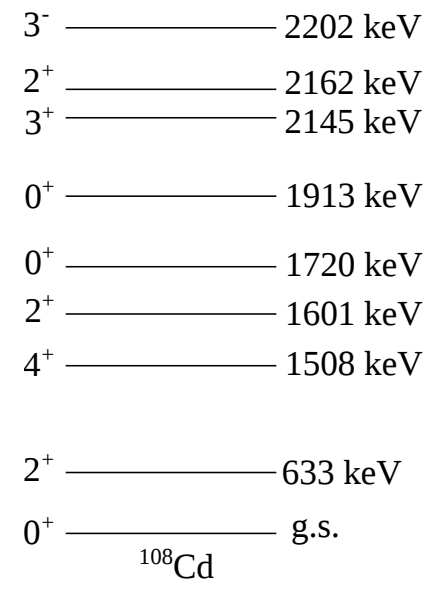

Figure 2. Levelscheme of ${ }^{108} \mathrm{Cd}$.

\section{Experimental method: The in-beam technique}

The proton beam was delivered by the $10 \mathrm{MV}$ FN tandem ion accelerator at the Institute for Nuclear Physics at the University of Cologne, Germany. The beam with an intensity of about $500 \mathrm{nA}$ impinged on silver targets with a $99.5 \%$ enrichment of ${ }^{107} \mathrm{Ag}$. Target thicknesses from $(2.70$ to 3.19$) \times 10^{18}$ atoms $/ \mathrm{cm}^{2}$ were determined by means of a Rutherford Backscattering Spectrometry measurement. The ${ }^{107} \mathrm{Ag}(\mathrm{p}, \gamma){ }^{108} \mathrm{Cd}$ reaction was studied by means of in-beam $\gamma$-ray spectroscopy at four different proton beam energies between $3.5 \mathrm{MeV}$ and $5.0 \mathrm{MeV}$ using the $\gamma$-ray spectrometer HORUS [4], which consists of 14 high-purity germanium (HPGe) detectors. The detectors are mounted under five different angles with respect to the beam axis which allows the measurement of angular distributions. The total detection efficiency of HORUS amounts to about $2 \%$ at an $\gamma$-ray energy of $E_{\gamma}=1.3 \mathrm{MeV}$. The angular distributions are fitted on a sum of Legendre polynomials using the function and used for the determination of absolute cross-section values.

$$
W(\Theta)=A_{0}\left(1+\sum_{k=2,4} \alpha_{k} P_{k}(\cos \Theta)\right)
$$

Those angular distributions are obtained for each ground state transition at each beam energy and the sum of all $\mathrm{A}_{0}$ coefficients represents the total number of reactions. The angular distribution for the $2_{1}^{+} \rightarrow$ g.s. transition at a beam energy of $E_{p}=5 \mathrm{MeV}$ is shown in Fig. 1.

Total cross sections are obtained by summing up all ground state transitions for each beam energy and partial cross sections are given by the direct population of a respective level in ${ }^{108} \mathrm{Cd}$. The $\mathrm{Q}$ value of this reaction amounts to $Q(p, \gamma)=8134 \mathrm{keV}$.

\section{Experimental results}

Not all of the seven observed ground-state transitions have the same intensity. The decay of the $2_{1}^{+}$ state at $E_{\gamma}=633 \mathrm{keV}$ has by far the highest contribution to the total ground state population with about $80 \%$. The contribution from the direct de-excitation of the compound state into the ground state, i.e. the $\gamma_{0}$, has a negligible contribution with about one percent. The experimentally obtained 


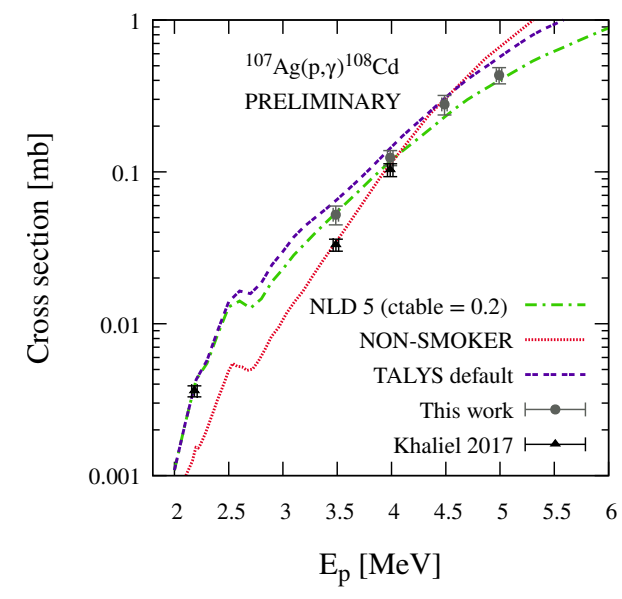

Figure 3. Total cross section results of the ${ }^{107} \mathrm{Ag}(\mathrm{p}, \gamma){ }^{108} \mathrm{Cd}$ reaction compared to statistical model calculations. The TALYS [5] default settings describe the data quite well, but overestimate the data slightly. The widely-used NON-SMOKER [9] code neither describes the energy trend nor the absolute values sufficiently well. Using the microscopic models for the $\gamma$-ray SF from Ref. [6] and the NLD from Ref. [7, 8] provides much better agreement with the experimental values. The microscopic NLD models have been slightly adjusted (see text for details) in order to obtain better agreement. For illustration only one adjusted NLD model is shown. The results from Khaliel et al. [10] do not completely agree with our results.

cross sections were compared to statistical model calculations using the TALYS code [5] in version 1.8. Various models have been used for the $\gamma$-ray strength function and nuclear level density. In detail, for the $\gamma$-ray strength function the TALYS $\gamma$-SF model 8 (Gogny D1M HFB+QRPA) [6] and for the nuclear level density (NLD) two microscopic models [7, 8] yielded excellent agreement with the experimental data. Both NLD models are based on a Hartree Fock Bogoliubov model. While the model from Ref. [7] is based on a zero-range Skyrme interaction the model from Ref [8] has the finiterange Gogny force implemented, which is more consistent with the $\gamma$-ray strength function model. As it is often used for microscopic models, called $\rho_{H F M}$, adjustment flexibility through a scaling function is provided within the TALYS code and applied for both NLD models for a better reproduction of the experimental values.

$$
\rho\left(E_{x}, J, \pi\right)=e^{c \cdot \sqrt{E_{x}-\delta}} \cdot \rho_{H F M}\left(E_{x}-\delta, J, \pi\right)
$$

By default $c=0$ and the 'pairing shift' $\delta$ is obtained from the cumulative number of low-lying levels. The experimental results of the total cross-section measurement as well as the comparison to theoretical calculations are shown in Fig. 3. Furthermore, recent results from Khaliel et al. [10] are shown, whereas they are systematically lower than our values. This might be due to the fact that they only take two ground-state transitions into account and assume that there is no angular dependence. However, Fig. 1 clearly shows that the latter is inevitable for the determination of absolute cross sections. Note, that both adjusted NLD models are able to describe the number of low-lying levels sufficiently well. This is a crucial demand each global nuclear level density model has to fulfil.

Partial cross sections up to the decay to the $8^{\text {th }}$ excited state at $E_{\gamma}=2202 \mathrm{keV}$ in ${ }^{108} \mathrm{Cd}$ have been observed in this experiment except for the $3^{+}$state at $E_{\gamma}=2145 \mathrm{keV}$. Partial cross sections are predominantly sensitive to the $\gamma$-ray strength function and according to the Brink-Axel hypothesis the $\gamma$-ray transmission probability is independent from the excitation energy. Therefore, the partial cross sections hold important information about the $\gamma$-ray strength function at the measured energies. It was found that no theoretical $\gamma$-ray strength function, neither phenomenological nor the microscopic was able to correctly describe the experimentally determined partial cross sections. Therefore, the microscopic dipole-strength function based on the Gogny D1M HFB+QRPA model has been adjusted individually for a better reproduction of the partial cross-section values. The resulting theoretical calculations for the default dipole strength as well as for the adjusted strength are shown in Fig. 4.

In summary, total and partial cross sections of the ${ }^{107} \mathrm{Ag}(\mathrm{p}, \gamma){ }^{108} \mathrm{Cd}$ reaction were measured by means of in-beam $\gamma$-ray spectroscopy. Using the total cross-section results slight adjustments on two microscopic NLD models could be performed in order to ensure an excellent agreement between 


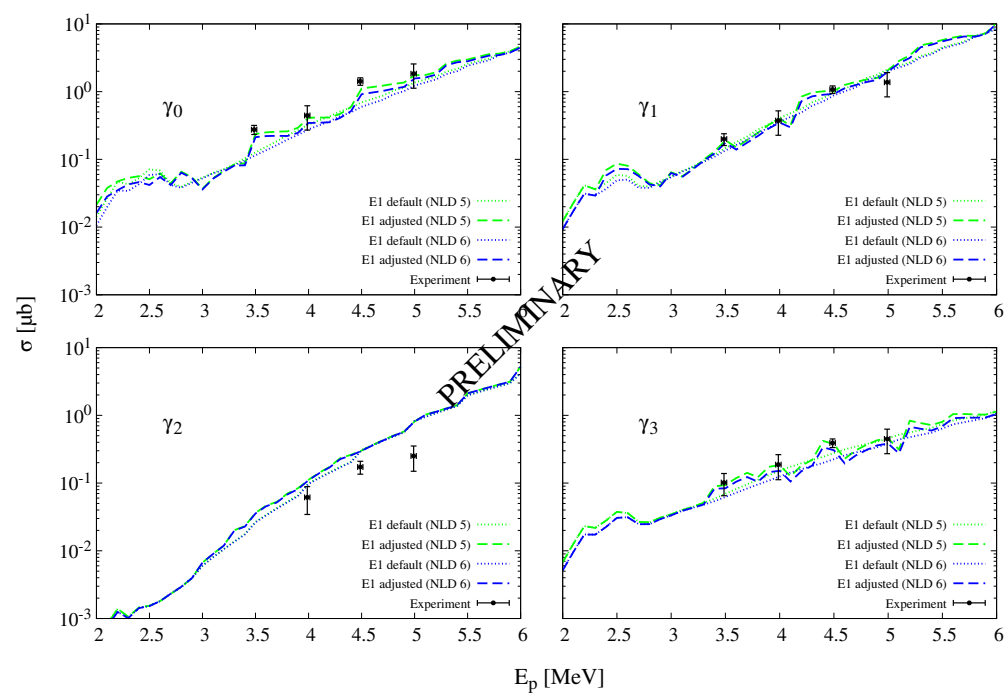

Figure 4. Experimentally determined partial cross sections compared to theoretical calculations. Both the default Gogny D1M HFB+QRPA $\gamma$-ray strength function as well as an adjusted one for a better reproduction of the experimental values has been used. Furthermore, both microscopic NLD models which have been adjusted via the total cross section measurement are used. Exemplary, partial cross sections for $\gamma_{0}$ to $\gamma_{3}$ transitions are shown.

experiment and theory. Furthermore, partial cross-section measurements were carried out and yielded an individual adjustment of the $\gamma$-ray strength function at $\gamma$-ray energies between $9.9 \mathrm{MeV}$ and 13.1 $\mathrm{MeV}$. Again, very good reproduction of the experimental results was achieved. As a next step, the total cross sections for beam energies of $2 \mathrm{MeV}$ and $2.7 \mathrm{MeV}$, for which experiments have been already performed, will be evaluated in order to verify the current finding at lower energies.

\section{Acknowledgement}

This project was supported by the Deutsche Forschungsgemeinschaft under Contract No. ZI 510/8-1 and the ULDETIS project within the University of Cologne Excellence Initiative strategy.

\section{References}

[1] M. Arnould and S. Goriely, Phys. Rep. 384, 1 (2003)

[2] T. Rauscher et al., Rep. Prog. Phys. 76, 066201 (2013)

[3] T. Rauscher, Phys. Rev. C 81, 045807 (2010)

[4] L. Netterdon et al., Nucl. Instr. Meth. A 758, 69 (2014)

[5] A. Koning, S. Hilaire, M. Duijvestijn, International Conf. on Nuclear Data, Nice, France (2007)

[6] M. Martini, S. Hilaire, S. Goriely, A.J. Koning and S. Péru, Nuclear Data Sheets 118, 273 (2014)

[7] S. Hilaire and S. Goriely Nucl. Phys., A 779, 63 (2006)

[8] S. Hilaire, M. Girod, S. Goriely and A.J. Koning, Phys. Rev. C 86, 064317 (2012)

[9] T. Rauscher and F.-K. Thielemann, Atomic Data and Nuclear Data Tables 79, 47 (2001)

[10] A. Khaliel et al., Phys. Rev. C 96, 035806 (2017) 